\title{
IMAGE
}

\section{Une érythrodermie typique}

\section{A typical erythrodermia}

Julien Bertolino ${ }^{a}$, Yasmine Beaussault ${ }^{b}$, Nolwenn Sautereau ${ }^{a}$, Audrey Benyamine ${ }^{a}$, Philippe Berbis $^{\mathrm{b}}$, Brigitte Granel ${ }^{\mathrm{a}}$,

${ }^{a}$ Service de Médecine Interne, Assistance Publique des Hôpitaux de Marseille (AP-HM), Hôpital Nord, Chemin des Bourrely, 13915 Marseille Cedex 15

${ }^{\mathrm{b}}$ Service de Dermatologie, Assistance Publique des Hôpitaux de Marseille (AP-HM), Hôpital Nord, Chemin des Bourrely, 13915 Marseille Cedex 15

Auteur correspondant :

Brigitte GRANEL

Service de médecine interne, hôpital Nord, chemin des Bourrely,

13915 Marseille cedex 15, France

Tél : 0491968712 Fax : 0491968080

Adresse e-mail: bgranel@ap-hm.fr

Mots clé : papulo-érythrodermie d'Ofuji ; érythrodermie; signe de la chaise longue Keywords : Ofuji's papuloerythroderma; erythroderma; deck chair sign 


\section{L'histoire}

Un homme de 70 ans, sans antécédents, consultait pour une dermatose érythémato-papuleuse prurigineuse généralisée évoluant depuis trois mois. Les premières lésions des avant-bras étaient survenues après avoir coupé des figuiers. Dans l'hypothèse d'une urticaire, un traitement par ébastine était proposé. L'atteinte cutanée s'aggravait et prédominait sur le tronc avec un aspect infiltré de la peau qui respectait les grands plis, notamment les plis abdominaux (Figure 1). On notait des adénopathies inguinales infra centimétriques.

La biologie retrouvait une hyperéosinophilie modérée (1,3 giga/L), une discrète lymphopénie, une augmentation modérée des IgE totales, sans syndrome inflammatoire. La recherche de cellules de Sézary circulantes, le typage lymphocytaire et l'électrophorèse des protéines sériques ne montraient pas d'anomalie. L'exploration immunologique retrouvait la présence isolée d'anticorps anti-nucléaires de fluorescence mouchetée (titre à 1/320) sans spécificité antigénique. L'histologie cutanée montrait une capillarite dermite à prédominance de polynucléaires éosinophiles non spécifique.

\section{Le diagnostic}

La papulo-érythrodermie d'Ofuji était diagnostiquée sur l'érythrodermie épargnant les plis cutanés, et notamment les grands plis de flexion et les plis cutanés abdominaux avec le signe de la « chaise longue », bien visible sur la Figure 1.

\section{Les commentaires}

La papulo-érythrodermie d'Ofuji se caractérise par des papules érythémateuses (voir brunâtres), prurigineuses dont la coalescence évolue rapidement vers une éruption étendue, touchant surtout le tronc, voire un tableau d'érythrodermie, respectant les grands plis de 
flexion, et notamment les plis cutanés abdominaux [1]. C'est une érythrodermie d'évolution chronique, avec présence d'adénopathies périphériques dans la moitié des cas, touchant le plus souvent des hommes d'âge mûr. Il existe une hyperéosinophilie dans plus de $80 \%$ des cas et une élévation des IgE totales dans $2 / 3$ des cas. Une lymphopénie est moins fréquente. L'histologie est non spécifique, mais permet d'exclure un lymphome ou une autre pathologie cutanée.

Dans la littérature, la papulo-érythrodermie d'Ofuji pourrait être associée à des cas d'hémopathies malignes, ainsi qu'à des néoplasies viscérales et notamment des cancers gastriques [2]. Chez le patient, un bilan exhaustif comprenant un scanner thoraco-abdominopelvien et une endoscopie oeso-gastro-duodénale, revenait négatif.

La corticothérapie locale, générale et la PUVA thérapie [3] sont les principaux traitements. Pour le patient, une corticothérapie locale a permis d'améliorer les lésions cutanées, mais un effet rebond a été observé à la décroissance de celle-ci. Dans la littérature, des traitements par ciclosporine, interféron alfa et azathioprine ont été proposés en cas de corticorésistance [4,5].

\section{Les auteurs ne déclarent aucun conflit d'intérêt.}




\section{Références}

[1] Torchia D, Miteva M, Hu S, Cohen C, Romanelli P. Papuloerythroderma 2009: two new cases and systematic review of the worldwide literature 25 years after its identification by Ofuji. Dermatology 2010;220:311-20.

[2] Teraki Y, Aso Y, Sato Y. High incidence of internal malignancy in papuloerythroderma of Ofuji: a case series from Japan. Dermatology 2012;224:5-9.

[3] Mutluer S, Yerebakan O, Alpsoy E, Ciftcioglu MA, Yilmaz E. Treatment of papuloerythroderma of Ofuji with Re-PUVA: a case report and review of the therapy. J Eur Acad Dermatol Venereol 2004;18:480-3.

[4] Jullien D, Kanitakis J, Souillet AL, Mathon N, Faure M, Claudy A. Ofuji's papuloerythroderma: treatment with cyclosporin A. Ann Dermatol Venereol 1998;125:589-92.

[5] Plantin P, Milochau P, Leroy JP, Berthou C, Sensebe L, Abgrall JF, et al. Ofuji's papuloerythroderma: efficacy of interferon-alpha. Ann Dermatol Venereol 1992;119:643-5. 


\section{Légende de la figure}

Figure 1 : Erythrodermie, épargnant les plis cutanés, et notamment les grands plis de flexion et les plis cutanés abdominaux avec le signe de la « chaise longue »

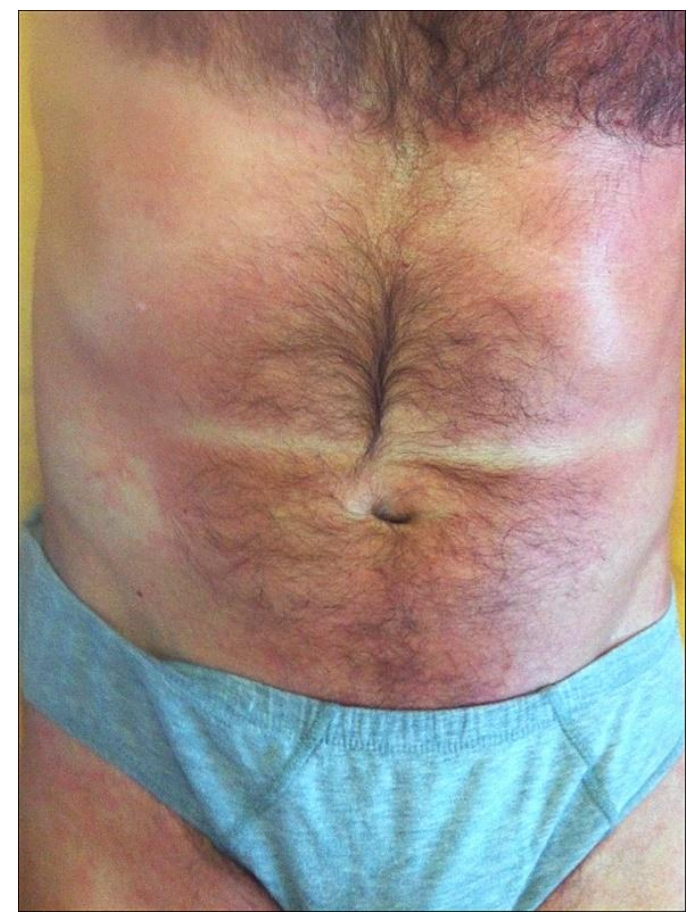

